\title{
Energy bounds for polycrystalline shape memory alloys
}

\author{
Michaël Peigney ${ }^{\mathrm{a}}$ \\ Laboratoire Central des Ponts et Chaussées, 58 Boulevard Lefebvre, 75732 Paris cedex 15, France
}

\begin{abstract}
In polycrystalline shape memory alloys at equilibrium under a prescribed loading, one typically observes the formation of austenite/martensite microstructures in each grain. The prediction of the resulting effective energy is a difficult question that still remains largely open. A related problem is the prediction of the set of recoverable (or zero-energy) strains of polycrystals. This article presents some recents results that have been achieved in that field, contrasting the geometrically linear theory ('infinitesimal strains') with the geometrically nonlinear theory ('finite strains').
\end{abstract}

\section{Introduction}

Consider a polycrystal of shape memory alloy, occupying a domain $\Omega$ in the reference configuration. A polycrystal consists in numerous monocrystalline grains with different orientations. The characteristic length of the domain $\Omega$ (macroscopic scale) is taken much larger than the typical length of the constitutive grains (mesoscopic scale). We decompose the domain $\Omega$ as $\Omega=\cup_{r=1}^{n} \Omega^{r}$ where each subdomain $\Omega^{r}$ is formed by grains having the same orientation. We denote by $\chi^{r}(\boldsymbol{x})$ the characteristic function of $\Omega^{r}$, defined by $\chi^{r}(\boldsymbol{x})=1$ if $\boldsymbol{x} \in \Omega^{r}$ and $\chi^{r}(\boldsymbol{x})=0$ if $\boldsymbol{x} \notin \Omega^{r}$.

The transformation of the crystal is described by a mapping $\boldsymbol{u}: \Omega \mapsto \mathbb{R}^{3}$. We study the equilibrium of the polycrystal under boundary conditions of the form $\boldsymbol{u}(\boldsymbol{x})=\overline{\boldsymbol{F}} \cdot \boldsymbol{x}$ where $\overline{\boldsymbol{F}}$ is given. Experimentally, the formation of austenite/martensite microstructures is observed in each grain, at a length scale much smaller thant the characteristic length scale of the grains. Three well-separated length scales are thus involved in the problem: the microscopic scale of the austenite/martensite microstructures, the mesoscopic scale of the constitutive grains, and the masoscopic scale of the polycrystal. Accordingly, the energy function takes different expressions at each scale. At the microscopic level, the free energy $\Psi^{r}$ at a point $\boldsymbol{x} \in \Omega^{r}$ is given by $\Psi^{r}(\boldsymbol{F})=\Psi^{0}\left(\boldsymbol{R}^{r, T} \boldsymbol{F} \boldsymbol{R}^{r}\right)$ where $\boldsymbol{F}=\nabla \boldsymbol{u}$ is the deformation gradient, $\boldsymbol{R}^{r}$ is a rotation, and $\Psi^{0}(\boldsymbol{F})$ is the microscopic energy of a reference single crystal. The function $\Psi^{0}$ is a multi well function of the form

$$
\Psi^{0}=\min _{1 \leq i \leq k} \Psi_{i}^{0}
$$

where $\Psi_{i}^{0}$ represents the microscopic energy of phase $i$. The mesoscopic energy function $Q \Psi^{r}$ in $\Omega^{r}$ is given by $Q \Psi^{r}(\boldsymbol{F})=Q \Psi^{0}\left(\boldsymbol{R}^{r, T} \boldsymbol{F} \boldsymbol{R}^{r}\right)$ where

$$
Q \Psi^{0}(\overline{\boldsymbol{F}})=\inf _{\boldsymbol{F} \in K(\overline{\boldsymbol{F}})} \frac{1}{|\Omega|} \int_{\Omega} \Psi^{0}(\boldsymbol{F}) d \boldsymbol{x}
$$

and

$$
K(\overline{\boldsymbol{F}})=\left\{\boldsymbol{F} \mid \exists \boldsymbol{u}: \Omega \mapsto \mathbb{R}^{3} \text { such that } \boldsymbol{F}=\nabla \boldsymbol{u} \text { in } \Omega, \text { and } \boldsymbol{u}=\overline{\boldsymbol{F}} \cdot \boldsymbol{x} \text { on } \partial \Omega\right\}
$$

\footnotetext{
a e-mail: michael.peigney@lcpc.fr
} 
At the macroscopic scale, the effective energy $\tilde{\Psi}$ is given by

$$
\tilde{\Psi}(\overline{\boldsymbol{F}})=\inf _{\boldsymbol{F} \in K(\overline{\boldsymbol{F}})} \frac{1}{|\Omega|} \int_{\Omega} \sum_{r=1}^{n} \chi^{r}(\boldsymbol{x}) Q \Psi^{r}(\boldsymbol{F}) d \boldsymbol{x}
$$

Whereas the microscopic energy function is assumed to be known, the problem of estimating $Q \Psi^{0}$ still remains largely open. That problem is directly connected to the prediction of the microstructures that develop in a reference single crystal. Estimating the macroscopic energy $\tilde{\Psi}$ of the polycrystal is even more challenging : stress and strain compatibility conditions between the grains have a direct influence of the selection of microstructures and the resulting effective energy. In this article, we are mostly concerned with deriving bounds on the effective energy $\tilde{\Psi}$. A related question is the prediction of the set $\tilde{\Pi}$ of energy-minimizing deformation gradients, as defined by $\tilde{\Pi}=\left\{\overline{\boldsymbol{F}} \mid \tilde{\Psi}(\overline{\boldsymbol{F}}) \leq \tilde{\Psi}\left(\overline{\boldsymbol{F}}^{\prime}\right) \forall \overline{\boldsymbol{F}}^{\prime}\right\}$. That set $\tilde{\Pi}$ plays a central role in the peculiar properties of shape memory alloys. Assuming - without loss of generality - that the minimum value of $\tilde{\Psi}$ is equal to 0 , we have

$$
\tilde{\Pi}=\{\overline{\boldsymbol{F}} \mid \tilde{\Psi}(\overline{\boldsymbol{F}})=0\}
$$

Let $\tilde{\Psi}_{-}$and $\tilde{\Psi}_{+}$be lower and upper bounds on $\tilde{\Psi}$. The inequality $\tilde{\Psi}_{-} \leq \tilde{\Psi} \leq \tilde{\Psi}_{+}$entails that $\left\{\overline{\boldsymbol{F}} \mid \tilde{\Psi}_{+}(\overline{\boldsymbol{F}}) \leq 0\right\} \subset \tilde{\Pi} \subset\left\{\overline{\boldsymbol{F}} \mid \tilde{\Psi}_{-}(\overline{\boldsymbol{F}}) \leq 0\right\}$. Therefore, a lower bound (resp. upper bound) on $\tilde{\Psi}$ translates into an upper bound (resp. lower bound) on $\tilde{\Pi}$, in the sense of inclusion of sets. Conversely, let $\tilde{\Pi}_{-}$and $\tilde{\Pi}_{+}$be lower and upper bounds on $\tilde{\Pi}$. From the relation $\tilde{\Pi}_{-} \subset \tilde{\Pi} \subset \tilde{\Pi}_{+}$, we simply obtain that $\tilde{\Psi}(\overline{\boldsymbol{F}})=0$ if $\overline{\boldsymbol{F}} \in \tilde{\Pi}_{-}, \tilde{\Psi}(\overline{\boldsymbol{F}})>0$ for $\overline{\boldsymbol{F}} \notin \tilde{\Pi}_{+}$. Bounds on $\tilde{\Pi}$ thus only provide limited information on $\tilde{\Psi}$. However, it turns out that bounding $\tilde{\Pi}$ is more tractable than bounding $\tilde{\Psi}$, especially in the geometrically nonlinear setting considered so far. The analysis is significantly simplified in the geometrically linear theory, assuming small perturbations with respect to the reference configuration. In that theory, the energy functions only depend on the deformation gradient $\boldsymbol{F}$ through the infinitesimal strain $\boldsymbol{e}=\frac{1}{2}\left(\boldsymbol{F}+\boldsymbol{F}^{T}\right)-\boldsymbol{I}$. In particular, the microscopic energy $\psi^{r}$ in $\Omega^{r}$ is written as $\psi^{r}(\boldsymbol{e})=\psi^{0}\left(\boldsymbol{R}^{r, T} \boldsymbol{e} \boldsymbol{R}^{r}\right)$ where $\psi^{0}(\boldsymbol{e})=\min _{1 \leq i \leq k} \psi_{i}^{0}(\boldsymbol{e})$. The mesoscopic energy function $Q \psi^{r}$ is given by $Q \psi^{r}(\boldsymbol{e})=Q \psi^{0}\left(\boldsymbol{R}^{r, T} \boldsymbol{e} \boldsymbol{R}^{r}\right)$ where

$$
Q \psi^{r}(\overline{\boldsymbol{e}})=\inf _{\boldsymbol{e} \in \mathcal{K}(\overline{\boldsymbol{e}})} \frac{1}{|\Omega|} \int_{\Omega} \psi^{r}(\boldsymbol{e}) d \boldsymbol{x}
$$

and

$$
\mathcal{K}(\overline{\boldsymbol{e}})=\left\{\boldsymbol{e} \mid \exists \boldsymbol{u}: \Omega \mapsto \mathbb{R}^{3} \text { such that } \boldsymbol{e}=\frac{1}{2}\left(\nabla \boldsymbol{u}+\nabla \boldsymbol{u}^{T}\right) \text { in } \Omega, \text { and } \boldsymbol{u}=\overline{\boldsymbol{e}} \cdot \boldsymbol{x} \text { on } \partial \Omega\right\}
$$

The effective energy $\tilde{\psi}$ is given by

$$
\tilde{\psi}(\overline{\boldsymbol{e}})=\inf _{\boldsymbol{e} \in \mathcal{K}(\overline{\boldsymbol{e}})} \frac{1}{|\Omega|} \int_{\Omega} \sum_{r=1}^{n} \chi^{r}(\boldsymbol{x}) Q \psi^{r}(\boldsymbol{e}) d \boldsymbol{x}
$$

The set of recoverable strains $\tilde{\pi}$ is

$$
\tilde{\pi}=\{\overline{\boldsymbol{e}} \mid \tilde{\psi}(\overline{\boldsymbol{e}})=0\}
$$

In this article, we primarly focus on lower bounds on the energy (thus corresponding to upper bounds on the energy-minimizing strains).

\section{Bounds in the geometrically linear theory}

In the geometrically linear theory of martensitic transformation, the microscopic free energy $\psi^{0}$ of the reference single crystal is generally taken in the form (1) with

$$
\psi_{i}^{0}(\boldsymbol{e})=\frac{1}{2}\left(\boldsymbol{e}-\boldsymbol{e}_{i}^{0}\right): \boldsymbol{L}^{0}:\left(\boldsymbol{e}-\boldsymbol{e}_{i}^{0}\right)+w_{i}^{0}
$$


The reference transformation strains $\boldsymbol{e}_{1}^{0}, \cdots, \boldsymbol{e}_{k}^{0}$ and the coefficients $w_{i}^{0}$ are given. For the sake of simplicity, we restrict our attention to isotropic tensors $\boldsymbol{L}^{0}$ (i.e. $L_{i j p q}^{0}=\lambda^{0} \delta_{i j} \delta_{p q}+\mu^{0}\left(\delta_{i p} \delta_{j q}+\right.$ $\left.\left.\delta_{i q} \delta_{j p}\right)\right)$.

\subsection{Bounds depending on one-point statistics}

Let $c^{r}=(1 /|\Omega|) \int_{\Omega} \chi^{r}$ be the volume fraction of orientation $r$. Combining convexification with the Sachs assumption of constant stress leads to the following lower bound on $\tilde{\psi}[2,3]$ :

$$
\tilde{\psi}(\overline{\boldsymbol{e}}) \geq \inf _{\overline{\boldsymbol{\theta}} \in \tilde{\mathcal{T}}} C \tilde{\psi}(\overline{\boldsymbol{e}}, \overline{\boldsymbol{\theta}})
$$

with

$$
C \tilde{\psi}(\overline{\boldsymbol{e}}, \overline{\boldsymbol{\theta}})=\frac{1}{2}(\overline{\boldsymbol{e}}-\boldsymbol{e}(\overline{\boldsymbol{\theta}})): \boldsymbol{L}^{0}:(\overline{\boldsymbol{e}}-\boldsymbol{e}(\overline{\boldsymbol{\theta}}))+\sum_{r, i} \bar{\theta}_{i}^{r} w_{i}^{0}
$$

and

$$
\tilde{\mathcal{T}}=\left\{\overline{\boldsymbol{\theta}} \in \mathbb{R}_{k}^{n} \mid \bar{\theta}_{i}^{r} \geq 0 ; \sum_{i=1}^{k} \bar{\theta}_{i}^{r}=c^{r} \forall r\right\}
$$

The tensor $\boldsymbol{e}(\overline{\boldsymbol{\theta}})$ in (11) is defined by $\boldsymbol{e}(\overline{\boldsymbol{\theta}})=\sum_{r, i} \bar{\theta}_{i}^{r} \boldsymbol{e}_{i}^{r}$ with $\boldsymbol{e}_{i}^{r}=\boldsymbol{R}^{r} \boldsymbol{e}_{i}^{0} \boldsymbol{R}^{r, T}$. The bound (10) depends on one-point statistics of the texture, i.e. on the volume fractions $c^{r}$ of the different orientations. Tighter first-order lower bounds have recently been obtained [7], making use of quasiconvex and quadratic comparison potentials. Recall that a function $\tilde{U}$ is quasiconvex if

$$
\tilde{U}(\overline{\boldsymbol{e}}) \leq \frac{1}{|\Omega|} \int_{\Omega} \tilde{U}(\boldsymbol{e}) d \boldsymbol{x} \text { for all } \overline{\boldsymbol{e}} \text { and } \boldsymbol{e} \in \mathcal{K}(\overline{\boldsymbol{e}})
$$

A fourth-order tensor $\boldsymbol{K}$ will be called quasiconvex if the quadratic function $\boldsymbol{e} \mapsto \frac{1}{2} \boldsymbol{e}: \boldsymbol{K}: \boldsymbol{e}$ satisfies (12). We will also use the notation $\boldsymbol{M}>0$ to indicate that a given fourth-order tensor $\boldsymbol{M}$ is positive definite, i.e. satisfies $\boldsymbol{e}: \boldsymbol{M}: \boldsymbol{e}>0$ for all $\boldsymbol{e} \neq 0$. Similarly, for a second-order tensor $\boldsymbol{a}$, the notation $\boldsymbol{a} \geq 0$ is used to indicate that $\boldsymbol{a}$ satisfies $\boldsymbol{u} . \boldsymbol{a} . \boldsymbol{u} \geq 0$ for all vector $\boldsymbol{u}$. The following inequality can be proved [7]:

$$
\tilde{\psi}(\overline{\boldsymbol{e}}) \geq \inf _{\overline{\boldsymbol{\theta}} \in \tilde{\mathcal{T}}} C \tilde{\psi}(\overline{\boldsymbol{e}}, \overline{\boldsymbol{\theta}})+G(\overline{\boldsymbol{\theta}})
$$

where $G$ is a positive function defined by

$$
G(\overline{\boldsymbol{\theta}})=\sup _{\boldsymbol{K} \in \mathcal{F} \mid \boldsymbol{L}^{0}-\boldsymbol{K}>0} \frac{1}{4} \sum_{r, s, i, j} \bar{\theta}_{i}^{r} \bar{\theta}_{j}^{s}\left(\boldsymbol{e}_{i}^{r}-\boldsymbol{e}_{j}^{s}\right): \boldsymbol{M}^{0}(\boldsymbol{K}):\left(\boldsymbol{e}_{i}^{r}-\boldsymbol{e}_{j}^{s}\right)
$$

In that expression, the tensor $\boldsymbol{M}^{0}(\boldsymbol{K})$ is defined by $\boldsymbol{M}^{0}(\boldsymbol{K})=\boldsymbol{L}^{0}-\boldsymbol{L}^{0}:\left(\boldsymbol{L}^{0}-\boldsymbol{K}\right): \boldsymbol{L}^{0}$, and $\mathcal{F}$ denotes an arbitrary family of quasiconvex tensors. For instance, such a family is formed by the functions $\boldsymbol{e} \mapsto-\boldsymbol{a}: \boldsymbol{e}^{*}$ where $\boldsymbol{a} \geq 0$ and $\boldsymbol{e}^{*}$ is the adjugate of $\boldsymbol{e}$ (defined by $e_{i i}^{*}=e_{j j} e_{k k}-e_{j k}^{2}$, $e_{j k}^{*}=e_{j i} e_{k i}-e_{j k} e_{i i}$ for any $(i, j, k)$ permutation of $\left.(1,2,3)\right)[6]$. Let $\boldsymbol{K}(\boldsymbol{a})$ be the symmetric fourth-order tensor such that $(1 / 2) \boldsymbol{e}: \boldsymbol{K}(\boldsymbol{a}): \boldsymbol{e}=-\boldsymbol{a}: \boldsymbol{e}^{*}$ for all $\boldsymbol{e}$. Using that particular class of quadratic quasiconvex functions in (14) and setting $\boldsymbol{M}^{0}(\boldsymbol{a})=\boldsymbol{L}^{0}-\boldsymbol{L}^{0}:\left(\boldsymbol{L}^{0}-\boldsymbol{K}(\boldsymbol{a})\right)^{-1}: \boldsymbol{L}^{0}$, we obtain that $\tilde{\psi}(\overline{\boldsymbol{e}}) \geq \inf _{\overline{\boldsymbol{\theta}} \in \tilde{\mathcal{T}}} C \tilde{\psi}(\overline{\boldsymbol{e}}, \overline{\boldsymbol{\theta}})+g(\overline{\boldsymbol{\theta}})$ where

$$
g(\overline{\boldsymbol{\theta}})=\sup _{\boldsymbol{a} \geq 0 \mid \boldsymbol{L}^{0}-\boldsymbol{K}(\boldsymbol{a})>0} \frac{1}{4} \sum_{r, s, i, j} \bar{\theta}_{i}^{r} \bar{\theta}_{j}^{s}\left(\boldsymbol{e}_{i}^{r}-\boldsymbol{e}_{j}^{s}\right): \boldsymbol{M}^{0}(\boldsymbol{a}):\left(\boldsymbol{e}_{i}^{r}-\boldsymbol{e}_{j}^{s}\right)
$$




\subsection{Bounds depending on two-point statistics}

The bounds considered so far only depend on the texture through the volume fractions $c^{r}$. Combining the translation method with a Hashin-Strikman variational formulation, it is actually possible to derive lower bounds incorporating two-point statistics. The corresponding bound takes a relatively simple expression in the case of textures with ellipsoidal symmetry. In such textures, the probability of finding orientation $r$ at point $\boldsymbol{x}$ and orientation $s$ at $\boldsymbol{x}^{\prime}$ is a function of $\mid \boldsymbol{Z}$. $\left(\boldsymbol{x}-\boldsymbol{x}^{\prime}\right) \mid$ where $\boldsymbol{Z}$ is a constant symmetric positive tensor. For any symmetric positive definite tensor $\tilde{\boldsymbol{L}}$, consider the fourth-order order tensor $\boldsymbol{P}[8]$ defined by

$$
\boldsymbol{P}=\frac{1}{4 \pi \operatorname{det} \boldsymbol{Z}} \int_{\|\boldsymbol{\xi}\|=1} \tilde{\boldsymbol{H}}(\boldsymbol{\xi})\left\|\boldsymbol{Z}^{-1} \cdot \boldsymbol{\xi}\right\|^{-3} d \boldsymbol{\xi}
$$

where $\tilde{\boldsymbol{H}}(\boldsymbol{\xi})$ is the fourth-order tensor with components $\tilde{H}(\boldsymbol{\xi})_{i j p q}=\xi_{j}\left[[\tilde{\boldsymbol{L}}(\boldsymbol{\xi})]^{-1}\right]_{i p} \xi_{q}$ and $\tilde{\boldsymbol{L}}(\boldsymbol{\xi})$ is the second-order tensor with components $\tilde{L}(\boldsymbol{\xi})_{i p}=\tilde{L}_{i j p q} \xi_{j} \xi_{q}$. The bound formula [7] is

$$
\tilde{\psi}(\overline{\boldsymbol{e}}) \geq \inf _{\overline{\boldsymbol{\theta}} \in \tilde{\mathcal{T}}} C \tilde{\psi}(\overline{\boldsymbol{e}}, \overline{\boldsymbol{\theta}})+H(\overline{\boldsymbol{\theta}})
$$

with

$$
\begin{aligned}
H(\overline{\boldsymbol{\theta}})= & \sup _{\tilde{\boldsymbol{K}} \in \mathcal{F}, \tilde{\boldsymbol{L}}_{>0 \mid} \boldsymbol{L}^{0}-\boldsymbol{K}>0}\left\{\frac{1}{4} \sum_{r, s, i, j} \bar{\theta}_{i}^{r} \bar{\theta}_{j}^{s}\left(\boldsymbol{e}_{i}^{r}-\boldsymbol{e}_{j}^{s}\right): \boldsymbol{M}^{0}(\boldsymbol{K}):\left(\boldsymbol{e}_{i}^{r}-\boldsymbol{e}_{j}^{s}\right)\right. \\
& \left.+\frac{1}{2} \sum_{r} \frac{1}{c_{r}} \boldsymbol{h}^{r}:\left(\boldsymbol{L}^{0}-\boldsymbol{K}+\left(\boldsymbol{L}^{0}-\boldsymbol{K}\right): \boldsymbol{P}:\left(\boldsymbol{L}^{0}-\boldsymbol{K}\right)\right)^{-1}: \boldsymbol{h}^{r}\right\}
\end{aligned}
$$

where $\boldsymbol{K}=\tilde{\boldsymbol{L}}+\tilde{\boldsymbol{K}}$ and $\boldsymbol{h}^{r}=\boldsymbol{L}^{0}:\left(c^{r} \boldsymbol{e}(\overline{\boldsymbol{\theta}})-\sum_{i=1}^{k} \bar{\theta}_{i}^{r} \boldsymbol{e}_{i}^{r}\right)$. Using the same class of quasiconvex tensors as in (15), we obtain that $\tilde{\psi}(\overline{\boldsymbol{e}}) \geq \inf _{\overline{\boldsymbol{\theta}} \in \tilde{\mathcal{T}}} C \tilde{\psi}(\overline{\boldsymbol{e}}, \overline{\boldsymbol{\theta}})+h(\overline{\boldsymbol{\theta}})$ with

$$
\begin{aligned}
h(\overline{\boldsymbol{\theta}})= & \sup _{\boldsymbol{a} \geq 0, \tilde{\boldsymbol{L}}>0 \mid \boldsymbol{L}^{0}-\boldsymbol{K}>0}\left\{\frac{1}{4} \sum_{r, s, i, j} \bar{\theta}_{i}^{r} \bar{\theta}_{j}^{s}\left(\boldsymbol{e}_{i}^{r}-\boldsymbol{e}_{j}^{s}\right): \boldsymbol{M}^{0}(\boldsymbol{a}):\left(\boldsymbol{e}_{i}^{r}-\boldsymbol{e}_{j}^{s}\right)\right. \\
& \left.+\frac{1}{2} \sum_{r} \frac{1}{c_{r}} \boldsymbol{h}^{r}:\left(\boldsymbol{L}^{0}-\boldsymbol{K}+\left(\boldsymbol{L}^{0}-\boldsymbol{K}\right): \boldsymbol{P}:\left(\boldsymbol{L}^{0}-\boldsymbol{K}\right)\right)^{-1}: \boldsymbol{h}^{r}\right\}
\end{aligned}
$$

\subsection{Example}

We consider a polycrystalline material, with two crystalline orientations in equal volume fractions. In a reference orthonormal basis $\left(\boldsymbol{v}_{1}, \boldsymbol{v}_{2}, \boldsymbol{v}_{3}\right)$, the two rotations $\boldsymbol{R}_{1}, \boldsymbol{R}_{2}$ defining the crystalline orientations are the identity matrix and the $\pi / 2$-rotation of axis $\boldsymbol{v}_{3}$. The microscopic energy $\psi^{0}$ of the reference single crystal is a three-well function given by $(1)(9)$ with $w_{1}^{0}=w_{2}^{0}=w_{3}^{0}=0, \boldsymbol{e}_{1}^{0}=\operatorname{diag}\left(\eta_{3}, \eta_{1}, \eta_{2}\right), \boldsymbol{e}_{2}^{0}=\operatorname{diag}\left(\eta_{2}, \eta_{3}, \eta_{1}\right), \boldsymbol{e}_{3}^{0}=\operatorname{diag}\left(\eta_{1}, \eta_{2}, \eta_{3}\right)$. The lattice parameters $\eta_{1}, \eta_{2}, \eta_{3}$ are taken as $\eta_{1}=0.06, \eta_{2}=0.02, \eta_{3}=-\eta_{1}-\eta_{2}$. For that particular material, the set $\tilde{\pi}$ of recoverable strains is included in the two-dimensional space of diagonal deviatoric tensors. The shaded domain on Figure 1 shows the upper bound on $\tilde{\pi}$ that is delivered by the energy bound (15), depending on one-point statistics. The green and red lines delimit the set of recoverable strains in the cases $c^{1}=0$ and $c^{1}=0$, i.e. in the homogeneous cases where only one orientation is present. The lower curve on Figure 1 (right) shows the values of the energy bound (15) for diagonal tensors $e_{11} \operatorname{diag}(1,1,-2)$ in the vicinity of $\left(\eta_{1}+\eta_{2}\right) / 2 \operatorname{diag}(1,1,-2)$. The upper curve of Figure 1 (right) shows the values of the energy bound (18), assuming an isotropic distribution of orientations 1 and 2. As expected, taking second-order statistics into account may significantly improve the estimation of the energy. 

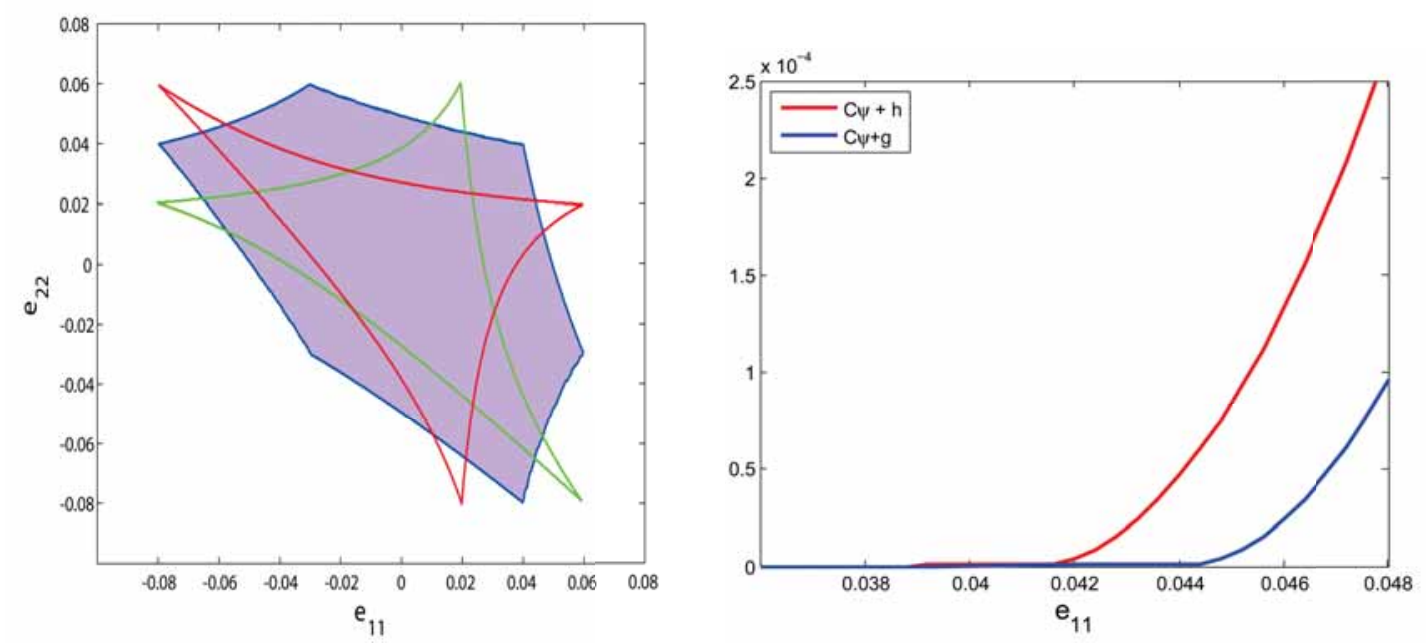

Fig. 1. (left) Upper bound on the recoverable strains for a three-well/two-orientation problem in the geometrically linear theory (right) Lower bounds on the effective energy.

\section{Bounds in the geometrically nonlinear theory}

\subsection{Bounds depending on one-point statistics}

Some of the bounding techniques presented in Section 2 can also be used in the geometrically nonlinear theory. However, the geometric nonlinearity significantly increases the difficulty of the analysis. At present stage, only bounds on the energy-minimizing strains have been studied [6]. More precisely, an upper bound $\Pi^{+}$on the set $\tilde{\Pi}$ is given by

$$
\Pi^{+}=\bigcap_{\boldsymbol{\tau}, \boldsymbol{a}, \alpha}\left\{\overline{\boldsymbol{F}} \mid 0 \geq \boldsymbol{\tau}: \overline{\boldsymbol{F}}+\boldsymbol{a}: \overline{\boldsymbol{F}}^{*}+\alpha \operatorname{det} \overline{\boldsymbol{F}}-\sum_{r=1}^{n} c^{r} f^{r}(\boldsymbol{\tau}, \boldsymbol{a}, \alpha)\right\}
$$

where

$$
f^{r}(\boldsymbol{\tau}, \boldsymbol{a}, \alpha)=\sup _{\boldsymbol{U} \in \boldsymbol{R}^{r, T} \mathcal{U}^{0} \boldsymbol{R}^{r}} \lambda_{1}+\lambda_{2}+\operatorname{sgn}(\operatorname{det} \boldsymbol{M}) \lambda_{3}+\alpha \operatorname{det} \boldsymbol{U}
$$

In (20), $\boldsymbol{M}$ is defined by $\boldsymbol{M}=\overline{\boldsymbol{\tau}} \boldsymbol{U}+\boldsymbol{a} \boldsymbol{U}^{*}$ and $\left(\lambda_{1}, \lambda_{2}, \lambda_{3}\right)$ are the singular values of the tensor $\operatorname{sgn}(\operatorname{det} \boldsymbol{M}) \boldsymbol{M}$, ordered in such a way that $\lambda_{1} \geq \lambda_{2} \geq \lambda_{3}$. The set $\mathcal{U}^{0}$ denotes the set of symmetric positive deformation gradients that are recoverable for the reference single crystal. It can be observed that the bound (19) only depends on one-point statistics. The derivation of bounds including higher-order statistical information remains to be investigated.

\subsection{Example}

The bound (19) is illustrated on a polycrystalline material with two orientations in equal volume fractions. In the reference basis $\left(\boldsymbol{v}_{1}, \boldsymbol{v}_{2}, \boldsymbol{v}_{3}\right)$, the rotations of the two orientations are the identity matrix and the $\pi / 4$-rotation of axis $\boldsymbol{v}_{3}$. The reference microscopic energy $\Psi^{0}$ has only two wells $\mathrm{SO}(3) \boldsymbol{U}_{1}^{0}$ and $\mathrm{SO}(3) \boldsymbol{U}_{2}^{0}$, where $\boldsymbol{U}_{1}^{0}$ and $\boldsymbol{U}_{2}^{0}$ are symmetric positive definite tensors verifying $\boldsymbol{U}_{i}^{0} \cdot \boldsymbol{v}_{3}=\boldsymbol{v}_{3}$ and $\operatorname{det} \boldsymbol{U}_{i}^{0}=1$ (for i=1,2). The tensors $\boldsymbol{U}_{1}^{0}$ and $\boldsymbol{U}_{2}^{0}$ are also assumed to be rank-1 connected, i.e. there exists a rotation $\boldsymbol{R}^{\prime}$ and two vectors $\boldsymbol{a}, \boldsymbol{n}$ such that $\boldsymbol{R}^{\prime} \boldsymbol{U}_{2}^{0}-\boldsymbol{U}_{1}^{0}=\boldsymbol{a} \otimes \boldsymbol{n}$. Under those assumptions, the set $\mathcal{U}^{0}$ defining the recoverable strains of the reference single crystal is known explicitly [1]. 


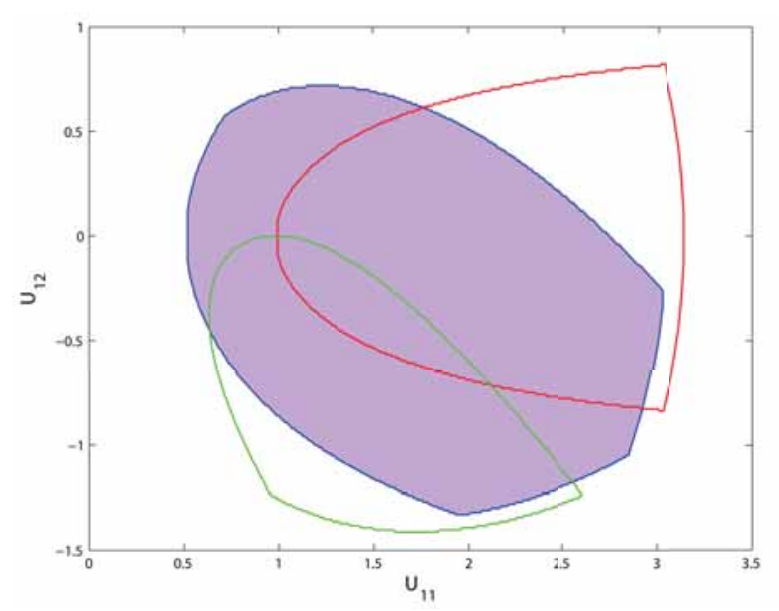

Fig. 2. Upper bound on the recoverable strains for a two-well/two-orientation problem in the geometrically nonlinear theory.

Consider an effective deformation gradient $\overline{\boldsymbol{F}}$, and let $\overline{\boldsymbol{U}}=\sqrt{\overline{\boldsymbol{F}}^{T} . \overline{\boldsymbol{F}}}$ be its right-stretch tensor. Because of the principle of frame indifference, $\overline{\boldsymbol{F}}$ is recoverable if and only if $\overline{\boldsymbol{U}}$ is recoverable. Moreover, for the problem considered, it is necessary for $\overline{\boldsymbol{U}}$ to be recoverable that $\overline{\boldsymbol{U}} \cdot \boldsymbol{v}_{3}=\boldsymbol{v}_{3}$ and $\operatorname{det} \overline{\boldsymbol{U}}=1$. Right-stretch tensors of recoverable deformation gradients are thus determined by their two components $\left(\bar{U}_{11}, \bar{U}_{12}\right)$. The dotted area on Figure 2 represents the bound (19), projected in the plane $\left(\bar{U}_{11}, \bar{U}_{12}\right)$. The red and blue lines delimit the set of recoverable right-stretch tensors in the homogeneous cases $c^{1}=1$ and $c^{1}=0$. The interpretation of Figure 2 is similar to that of Figure 1 (left) in the geometrically linear theory. However, in the geometrically nonlinear theory, we do not have results analog to Figure 1 (right): a strictly positive lower bound on the energy is not available.

\section{Concluding remarks}

In this article have been presented some recent lower bounds on the effective energy of polycrystalline shape-memory alloys. A common point to those bounds is the essential role played by the translation method [4]. In the geometrically linear theory, energy bounds depending on two-point statistics have been obtained. In contrast, in the geometrically nonlinear theory, only bounds on the zero-set of the energy, depending on one-point statistics, have been studied so far. Clearly, some progress remains to be made in the geometrically nonlinear theory. Even in the geometrically linear theory, it would be interesting to investigate if three- or higherpoint statistics could be taken into account, for instance using techniques presented in [5]. The attainabilty of the bounds obtained is another question worthy of interest.

\section{References}

1. Ball J.M., James R.D., Phil.Trans.Roy.Soc.London A. 338, (1992) p.338-450

2. Govindjee S., Mielke A., Hall G.J., J.Mech.Phys.Solids 51, (2003) p.I-XXVI

3. Hackl K., Heinen R., Schmahl W.W., Hasan M., Mat.Sci.Eng.A 481, (2007) p.347-350

4. Milton G., The theory of composites (Cambridge University Press, 2002)

5. Peigney M., J.Mech.Phys.Solids 53, (2005) p.923-948

6. Peigney M., J.Mech.Phys.Solids 56, (2008) p.360-375

7. Peigney M., J.Mech.Phys.Solids 57, (2009) p.970-986

8. Willis J.R., Adv.Appl.Mech. 21, (1981) p.1-78 